\title{
An Analysis of Eccentricity-Based Invariants for Biochemical Hypernetworks
}

\author{
Muhammad Aamer Rashid (D), Sarfraz Ahmad (D, ${ }^{1}$ Muhammad Kamran Siddiqui (iD, 1 \\ Shazia Manzoor $\left({ }^{\circ},{ }^{1}\right.$ and Mlamuli Dhlamini $\mathbb{1}^{2}$ \\ ${ }^{1}$ Department of Mathematics, COMSATS University Islamabad, Lahore Campus, Lahore, Pakistan \\ ${ }^{2}$ Department of Applied Mathematics, National University of Science and Technology, Bulawayo, Zimbabwe \\ Correspondence should be addressed to Mlamuli Dhlamini; mlamuli.dhlamini@nust.ac.zw
}

Received 25 August 2021; Accepted 6 October 2021; Published 18 October 2021

Academic Editor: Sampath Pradeep

Copyright (C) 2021 Muhammad Aamer Rashid et al. This is an open access article distributed under the Creative Commons Attribution License, which permits unrestricted use, distribution, and reproduction in any medium, provided the original work is properly cited.

\begin{abstract}
Biological proceedings are well characterized by solid illustrations for communication networks. The framework of biological networks has to be considered together with the expansion of infectious diseases like coronavirus. Also, the graph entropies have established themselves as the information theoretic measure to evaluate the architectural information of biological networks. In this article, we examined conclusive biochemical networks like $t$-level hypertrees along with the corona product of hypertrees with path. We computed eccentricity-based indices for the depiction of aforementioned theoretical frameworks of biochemical networks. Furthermore, explicit depiction of the graph entropies with these indices is also measured.
\end{abstract}

\section{Introduction}

Chemical graph theory is the developed mathematical field to study the problems of chemical networks. This field has extensive applications in computer sciences, mathematics, sociology, biology, medicine, and physics $[1,2]$. The biological entities such as proteins, RNA, DNA, metabolites, and graphs are used to grab the association between these entities $[3,4]$. Topological analysis of wide-ranging protein association network can bring intuition into repetition which can be used to predict protein functions [5, 6]. Furthermore, regulation approaches for contagious diseases generally rely on graph theoretic networks [7]. Schnitzler and Grass investigated initial analysis of neurological [8].

The obtained results are applied for decision control measures [9]. Kucharski et al. [10] investigated the fluctuations in transmission rates to analyze the efficiency of the control measures. Roosa et al. [11] illustrated phenomenological models to anticipate the dynamic of COVID-19. In [12], artificial intelligence approach is presented to find the top-quality prognostic models for the investigation of infectious diseases. Wan et al. [13] estimated risk-recognition for doubtful COVID-19 cases with the help of the graph embedding method. These complex networks have key role in communication systems, Internet, the World Wide Web, environment, and public health. Due to the extensive applications of complex networks, epidemiological and ecological researchers have chased their consideration to network analysis.

A tree in which one vertex has been nominated as the root and each edge is extended away from root is known as hyper tree. Different biological organisms like DNA sequences or different species could be represented by the vertices of a rooted tree. Aforementioned rooted trees of biological concern are termed as evolutionary trees of phytogenetic trees. A hypertree is a network topology and is a mixture of the hypercube concept and the binary tree. Artificial intelligence and machine learning approaches have demonstrated that when contact tracing is comprehensively exercised, one can alleviate the outbreak of the pandemic by cracking the existing sequence of spread of the coronavirus and consequently supporting to decrease the rate of current epidemic [14]. 
Consider $\mathscr{G}=\left(\mathscr{G}_{V}, \mathscr{G}_{E}\right)$ be a graph in which $\mathscr{G}_{V}$ and $\mathscr{G}_{E}$ are used to represent the vertex set and the edge set of $\mathscr{G}$, respectively. The degree of any vertex $l$ is termed as the number of edges associated to it and is denoted by $\wp(l)$. The maximum distance between $l$ and any other vertex of $\mathscr{G}$ is termed as eccentricity of $l$ and is denoted by $\varrho(l)$. Also, if $l m \in \mathscr{G}_{E}$, then $\wp(l)$ and $\wp(m)$ denote the degrees of vertex $l$ and $m$, respectively. In QSPR/QSAR studies, a lot of molecular descriptors are employed to correlate different biological and physico-chemical activities. In this study, we will talk about some eccentricity-based and degree-based indices.

Uncertainty is prevailing. It turns up as a consequence of insufficient information than the whole information required to identify its circumferences. In 1948, Shannon [15] established a criterion to guess the uncertainty identified as entropy. The entropy measure has identified comprehensive employment in physical sciences [16]. In the literature, numerous graph entropies are estimated by eccentricity of the vertices and characteristic polynomials [17]. Manzoor et al. talked about few relations between the complexity of graphs and Hosoya entropy $[18,19]$.

In 2014, Chen et al. [17] established the description of the entropy in equation (1) as follows:

$$
\operatorname{ENT}_{\psi}(\mathscr{G})=-\sum_{l^{\prime} m^{\prime} \in \mathscr{G}_{E}} \frac{\psi\left(l^{\prime} m^{\prime}\right)}{\sum_{l m \in \mathscr{G}_{E}} \psi(l m)} \log \left[\frac{\psi\left(l^{\prime} m^{\prime}\right)}{\sum_{l m \in \mathscr{G}_{E}} \psi(l m)}\right] .
$$

Also, the compact form of some eccentricity-based topological indices is depicted in Table 1, and some degreebased topological indices are depicted in Table 2.

\section{Methodology}

To enumerate our findings, we will exert the approach of combinatorial computing, edge partition technique, analytic methods, degree enumerating technique, and graph theoretic tools. Additionally, we will utilize Matlab and maple software for mathematical computing. For plotting our obtained results, we will use Microsoft Excel.

\section{Structure of Complete Hypertree}

A complete binary tree is termed as hypertree; we will denote it by $\mathscr{C} \mathscr{B} \mathscr{T}(t)$ with $t$ levels where level $k, 0 \leq k \leq t$, includes $2^{k}$ vertices. The vertices of $\mathscr{C} \mathscr{B} \mathscr{T}(t)$ are designated in the following way: the label of root node is 1 and it is at level 0 . For any vertex $l$, the children of $l$ are tagged with $2 l$ and $2 l+1$ [28]. In a hypertree, extra edges are horizontal, where in the same level $k, 1 \leq k \leq t$, any two vertices are attached by an edge (see Figure 1). Consider the hypertree $\mathscr{C} \mathscr{B} \mathscr{T}(3)$ in Figure 1 (see [28]) as an illustration to deduce distinct topological indices and their respective entropies. To demonstrate our main findings, we form a partition of edges of the hypertree $\mathscr{C} \mathscr{B} \mathscr{T}(t)$ for $t$ levels established on eccentricity of end vertices in Tables 3 and 4 representing the edge partition of $\mathscr{C} \mathscr{B} \mathscr{T}(t)$.

The quantitative structure activity relationship research of dendrimers could then be assisted by the distinct topological indices and their respective entropies acquired in this study for hypertrees [29]. In addition, current development of topological indices examined in [30] has substantial consequences in complex networks of material and molecular systems in which larger atoms and many other huge elements are presented [31]. For such systems, the relativistic consequences are very significant.

3.1. Eccentricity-Based Entropies of $\mathscr{C} \mathscr{B} \mathscr{T}(t)$. In this segment, we measure the eccentricity-based entropies of the complete hypertree $\mathscr{C} \mathscr{B} \mathscr{T}(t)$.

3.1.1. The Fourth Geometric Arithmetic Eccentric Entropy. Now, using Tables 1 and 3, the fourth geometric arithmetic eccentric index is calculated in [28] as follows:

$$
G A_{4}(\mathscr{C} \mathscr{B} \mathscr{T})=1+2^{t}+\sum_{k=1}^{t-1} 2^{k+2}\left(\frac{\sqrt{\left(t^{2}+k^{2}+2 t k-t-k\right)}}{2(t+k)-1}\right) \text {. }
$$

We computed $\mathrm{ENT}_{\mathrm{GA}_{4}}$ as follows:

$$
\begin{aligned}
& \operatorname{ENT}_{G A_{4}}(\mathscr{C} \mathscr{B} \mathscr{T})=\log \left(G A_{4}\right)-\frac{1}{\left(G A_{4}\right)} \sum_{i=1}^{3} \sum_{l m \in E_{i}(\mathscr{C} \mathscr{B} \mathscr{T})}\left[\frac{2 \sqrt{\varrho(l) \varrho(m)}}{\varrho(l)+\varrho(m)}\right] \log \left[\frac{2 \sqrt{\varrho(l) \varrho(m)}}{\varrho(l)+\varrho(m)}\right], \\
& \operatorname{ENT}_{G A_{4}}(\mathscr{C} \mathscr{B} \mathscr{T}(t))=\log \left(1+2^{t}+\sum_{k=1}^{t-1} 2^{k+2}\left(\frac{\sqrt{\left(-t+2 t k+t^{2}+k^{2}-k\right)}}{2(t+k)-1}\right)\right) \\
& -\frac{\sum_{k=1}^{t-1} 2^{k+2}\left(\sqrt{\left(t^{2}+k^{2}+2 t k-t-k\right)} / 2(t+k)-1\right) \log \left[\sum_{k=1}^{t-1} 2^{k+2}\left(\sqrt{\left(t^{2}+k^{2}+2 t k-t-k\right)} / 2(t+k)-1\right)\right]}{\left(1+2^{t}+\sum_{k=1}^{t-1} 2^{k+2}\left(\sqrt{\left(t^{2}+k^{2}+2 t k-t-k\right)} / 2(t+k)-1\right)\right)} . \\
& M M_{1}(\mathscr{C} \mathscr{B} \mathscr{T})=(12 t-14) \times 2^{t}-6 t+16 .
\end{aligned}
$$

3.1.2. The First Zagreb Eccentric Entropy. The first Zagreb eccentric index by using Tables 1 and 3 is calculated in [28] as follows:
We computed $\mathrm{ENT}_{M M_{1}}$ as follows: 
TABLE 1: Eccentricity-based topological indices along with their respective edge weight $\psi(\operatorname{lm})$ of the edge $l m$.

\begin{tabular}{lcc}
\hline Eccentricity-based topological indices & Edge weight $\psi(l m)$ of the edge $l m$ & Entropies \\
\hline The fourth geometric arithmetic eccentricity index $[20]$ & $(2 \sqrt{\varrho(l) \varrho(m)} /(\varrho(l)+\varrho(m)))$ & The fourth geometric arithmetic eccentricity \\
entropy & The first Zagreb eccentric entropy \\
The first Zagreb eccentric index [20] & $\varrho(l)+\varrho(m)$ & The second Zagreb eccentric entropy \\
The second Zagreb eccentric index [20] & $\varrho(l) \cdot \varrho(m)$ & Eccentric atom bond connectivity entropy \\
Eccentric atom bond connectivity index [21] & $\sqrt{(\varrho(l)+\varrho(m)-2) /(\varrho(l) \times \varrho(m))}$
\end{tabular}

TABLE 2: Degree-based topological indices along with their respective edge weight $\psi(l m)$ of the edge $l m$.

\begin{tabular}{|c|c|c|}
\hline Degree-based topological indices & Edge weight $\psi(\mathrm{lm})$ of the edge $l \mathrm{~m}$ & Entropies \\
\hline The hyper Zagreb index [22] & {$[\wp(l)+\wp(m)]^{2}$} & The hyper Zagreb entropy [23-25] \\
\hline The forgotten index [26] & $(\wp(l))^{2}+(\wp(m))^{2}$ & The forgotten entropy [23-25] \\
\hline The atom bond connectivity index [27] & $\sqrt{((\wp(l)+\wp(m)-2) /(\wp(l) \times \wp(m)))}$ & The atom bond connectivity entropy $[19,23,24]$ \\
\hline
\end{tabular}

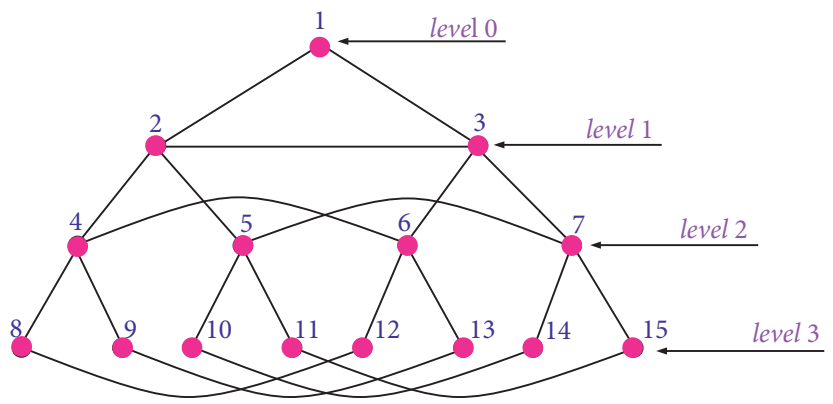

FIgUre 1: An illustration of the hypertree $\mathscr{C} \mathscr{B} \mathscr{T}(t)$ for $t=3$ levels [28].

TABLE 3: Edge partition of $\mathscr{C} \mathscr{B} \mathscr{T}(t)$ for $t$-levels.

\begin{tabular}{lcr}
\hline$(\varrho(l), \varrho(m))$ & Number of repetition & Range of $t$ and $k$ \\
\hline$(t, t)$ & 3 & $t \geq 3$ \\
$(t+k-1, t+k)$ & $2^{k+1}$ & $t \geq 3,1 \leq k \leq t-1$ \\
$(t+k, t+k)$ & $2^{k}$ & $t \geq 3,1 \leq k \leq t-1$ \\
\hline
\end{tabular}

TABLe 4: Edge partition of $\mathscr{C} \mathscr{B} \mathscr{T}(t)$ with $t$-levels.

\begin{tabular}{lcc}
\hline$(\wp(l), \wp(m))$ & Number of repetition & Kinds of edges \\
\hline$(2,2)$ & $2^{t-1}$ & $E_{1}$ \\
$(2,4)$ & 2 & $E_{2}$ \\
$(4,2)$ & $2^{t}$ & $E_{3}$ \\
$(4,4)$ & $2^{t+1}-2^{t-1}-5$ & $E_{4}$ \\
\hline
\end{tabular}




$$
\begin{aligned}
\operatorname{ENT}_{M M_{1}}(\mathscr{C} \mathscr{B} \mathscr{T})= & \log \left(M M_{1}\right)-\frac{1}{\left(M M_{1}\right)} \sum_{i=1}^{3} \sum_{l m \in E_{i}(\mathscr{C} \mathscr{B} \mathscr{T})}[\varrho(l)+\varrho(m)] \log [\varrho(l)+\varrho(m)], \\
\mathrm{ENT}_{M M_{1}}(\mathscr{C} \mathscr{B} \mathscr{T})= & \log \left((12 t-14) \times 2^{t}-6 t+16\right)-\frac{6 t \log [2 t]}{\left((12 t-14) \times 2^{t}-6 t+16\right)} \\
& -\frac{\sum_{k=1}^{t-1} 2^{k+1}(2 k+2 t-1) \log \left[\sum_{k=1}^{t-1}(2 k+2 t-1)\right]}{\left((12 t-14) \times 2^{t}-6 t+16\right)}-\frac{\sum_{k=1}^{t-1} 2^{k}(2 k=2 t) \log \left[\sum_{k=1}^{t-1}(2 k+2 t)\right]}{\left((12 t-14) \times 2^{t}-6 t+16\right)} \\
= & \log \left((12 t-14) \times 2^{t}-6 t+16\right)-\frac{6 t \log [2 t]}{\left((12 t-14) \times 2^{t}-6 t+16\right)} \\
& -\frac{\left((8 t-10) 2^{t}-8 t+12\right) \log \left[3 t^{2}-4 t+1\right]}{\left((12 t-14) \times 2^{t}-6 t+16\right)}-\frac{\left((4 t-4) 2^{t}-4 t+4\right) \log [(3 t / 2)(t-1)]}{\left((12 t-14) \times 2^{t}-6 t+16\right)} .
\end{aligned}
$$

3.1.3. The Second Zagreb Eccentric Entropy. The second $M M_{2}(\mathscr{C} \mathscr{B} \mathscr{T})=\left(12 t^{2}-28 t+22\right) \times 2^{t}-3 t^{2}+16 t-22$. Zagreb eccentric index by using Tables 1 and 3 is calculated in [28] as follows:

We computed $\mathrm{ENT}_{\mathrm{MM}_{2}}$ as follows:

$$
\begin{aligned}
\operatorname{ENT}_{M M_{2}}(\mathscr{C} \mathscr{B} \mathscr{T})= & \log \left(M M_{2}\right)-\frac{1}{\left(M M_{2}\right)} \sum_{i=1}^{3} \sum_{l m \in E_{i}(\mathscr{C} \mathscr{B} \mathscr{T})}[\varrho(l)+\varrho(m)] \log [\varrho(l)+\varrho(m)], \\
\operatorname{ENT}_{M M_{2}}(\mathscr{C} \mathscr{B} \mathscr{T}(t))= & \log \left(\left(12 t^{2}-28 t+22\right) \times 2^{t}-3 t^{2}+16 t-22\right)-\frac{3 t^{2} \log \left[t^{2}\right]}{\left(\left(12 t^{2}-28 t+22\right) \times 2^{t}-3 t^{2}+16 t-22\right)} \\
& -\frac{\sum_{k=1}^{t-1} 2^{k+1}\left(\left(t^{2}+k^{2}+2 t k-t-k\right)\right) \log \left[\sum_{k=1}^{t-1}\left(\left(t^{2}+k^{2}+2 t k-t-k\right)\right)\right]}{\left(\left(12 t^{2}-28 t+22\right) \times 2^{t}-3 t^{2}+16 t-22\right)} \\
& -\frac{\left(\left(12 t^{2}-28 t+22\right) \times 2^{t}-3 t^{2}+16 t-22\right)}{\left(\sum_{k=1}^{t-1} 2^{k}(t+k)^{2} \log \left[\sum_{k=1}^{t-1}(t+k)^{2}\right]\right.} \\
= & \log \left(\left(12 t^{2}-28 t+22\right) \times 2^{t}-3 t^{2}+16 t-22\right)-\frac{3 t^{2} \log \left[t^{2}\right]}{\left(\left(12 t^{2}-28 t+22\right) \times 2^{t}-3 t^{2}+16 t-22\right)} \\
& -\frac{\left(\left(8 t^{2}-20 t+16\right) 2^{t}-4 t^{2}+12 t-16\right) \log \left[(7 / 3) t^{3}-4 t^{2}+(5 / 3) t\right]}{\left(\left(12 t^{2}-28 t+22\right) \times 2^{t}-3 t^{2}+16 t-22\right)} \\
& -\frac{\left(\left(4 t^{2}-8 t+6\right) 2^{t}-2 t^{2}+4 t-6\right) \log \left[(7 / 3) t^{3}-(5 / 2) t^{2}+(1 / 6) t\right]}{\left.\left(12 t^{2}-28 t+22\right) \times 2^{t}-3 t^{2}+16 t-22\right)}
\end{aligned}
$$

3.1.4. Eccentric Atom Bond Connectivity Entropy. The eccentric atom bond connectivity index by using Tables 1 and 3 is calculated in [28] as follows:

$$
A B C_{5}(\mathscr{C} \mathscr{B} \mathscr{T})=\frac{3 \sqrt{2 t-2}}{t}+\sum_{k=1}^{t-1} 2^{k+1} \sqrt{\frac{2 t+2 k-3}{(t+k-1)(t+k)}}+\sum_{k=1}^{t-1} 2^{k \sqrt{2(t+k)-2}}
$$


We computed $\mathrm{ENT}_{A B C_{5}}$ as follows:

$$
\begin{aligned}
\operatorname{ENT}_{A B C_{5}}(\mathscr{C} \mathscr{B} \mathscr{T})= & \log \left(A B C_{5}\right)-\frac{1}{\left(A B C_{5}\right)} \sum_{i=1}^{3} \sum_{l m \in E_{i}(\mathscr{C} \mathscr{B})}\left[\sqrt{\frac{\varrho(l)+\varrho(m)-2}{\varrho(l) \times \varrho(m)}}\right] \log \left[\sqrt{\left.\frac{\varrho(l)+\varrho(m)-2}{\varrho(l) \times \varrho(m)}\right]},\right. \\
\mathrm{ENT}_{A B C_{5}}((\mathscr{C} \mathscr{B} \mathscr{T}(t))= & \log \left(\frac{3 \sqrt{2 t-2}}{t}+\sum_{k=1}^{t-1} 2^{k+1} \sqrt{\frac{2 t+2 k-3}{(t+k-1)(t+k)}}+\sum_{k=1}^{t-1} 2^{k} \frac{\sqrt{2(t+k)-2}}{t+k}\right) \\
& -\frac{(((3 \sqrt{2 t-2}) / t) \log [\sqrt{2 t-2} / t]}{\left.((3 \sqrt{2 t-2}) / t)+\sum_{k=1}^{t-1} 2^{k+1} \sqrt{(2 t+2 k-3) /\left(t^{2}+k^{2}+2 t k-t-k\right)}+\sum_{k=1}^{t-1} 2^{k}(\sqrt{2(t+k)-2} /(t+k))\right)} \\
& -\frac{\sum_{k=1}^{t-1} 2^{k+1} \sqrt{(2 t+2 k-3) /\left(t^{2}+k^{2}+2 t k-t-k\right)} \log \left[\sum_{k=1}^{t-1} \sqrt{(2 t+2 k-3) /\left(t^{2}+k^{2}+2 t k-t-k\right)}\right]}{\left(((3 \sqrt{2 t-2}) / t)+\sum_{k=1}^{t-1} 2^{k+1} \sqrt{(2 t+2 k-3) /\left(t^{2}+k^{2}+2 t k-t-k\right)}+\sum_{k=1}^{t-1} 2^{k}(\sqrt{2(t+k)-2} /(t+k))\right)} \\
& -\frac{\sum_{k=1}^{t-1} 2^{k}(\sqrt{2(t+k)-2} /(t+k)) \log \left[\sum_{k=1}^{t-1}(\sqrt{2(t+k)-2} /(t+k))\right]}{\left(((3 \sqrt{2 t-2}) / t)+\sum_{k=1}^{t-1} 2^{k+1} \sqrt{(2 t+2 k-3) /\left(t^{2}+k^{2}+2 t k-t-k\right)}+\sum_{k=1}^{t-1} 2^{k}(\sqrt{2(t+k)-2} /(t+k))\right)}
\end{aligned}
$$

3.2. Degree-Based Entropies of $\mathscr{C} \mathscr{B} \mathscr{T}(t)$. In this segment, we measure the degree-based entropies of the hypertree $\mathscr{C} \mathscr{B} \mathscr{T}(t)$.
3.2.1. The Hyper Zagreb Entropy. Now, using Tables 1 and 4, the hyper Zagreb index is calculated in [28] as follows:

$$
\operatorname{HM}(\mathscr{C} \mathscr{B} \mathscr{T})=104 \times 2^{t}-248 .
$$

We computed $\mathrm{ENT}_{\mathrm{HM}}$ as follows:

$$
\begin{aligned}
& \mathrm{ENT}_{\mathrm{HM}}(\mathscr{C} \mathscr{B} \mathscr{T})=\log (\mathrm{HM})-\frac{1}{(\mathrm{HM})} \sum_{i=1}^{4} \sum_{l m \in E_{i}(\mathscr{C} \mathscr{B} \mathscr{T})}[\wp(l)+\wp(m)]^{2} \log \left([\wp(l)+\wp(m)]^{2}\right), \\
& \mathrm{ENT}_{\mathrm{HM}}(\mathscr{C} \mathscr{B} \mathscr{T}(t))=\log \left(104 \times 2^{t}-248\right)-\frac{2^{t+3} \log [16]}{\left(104 \times 2^{t}-248\right)}-\frac{36\left(2+2^{t}\right) \log [36]}{\left(104 \times 2^{t}-248\right)}-\frac{\left(2^{t+7}-2^{t+5}-320\right) \log [64]}{\left(104 \times 2^{t}-248\right)} . \\
& F(\mathscr{C} \mathscr{B} \mathscr{T})=76 \times 2^{t}-120 .
\end{aligned}
$$

3.2.2. The Forgotten Entropy. Now, using Tables 1 and 4, the forgotten index is calculated in [28] as follows:
We computed $\mathrm{ENT}_{F}$ as follows:

$$
\begin{aligned}
& \operatorname{ENT}_{F}(\mathscr{C} \mathscr{B} \mathscr{T})=\log (F)-\frac{1}{(F)} \sum_{i=1}^{4} \sum_{l m \in E_{i}(\mathscr{C} \mathscr{B})}\left[(\wp(l))^{2}+(\wp(m))^{2}\right] \log \left[((l))^{2}+(\wp(m))^{2}\right], \\
& \operatorname{ENT}_{F}(\mathscr{C} \mathscr{B} \mathscr{T})=\log \left(76 \times 2^{t}-120\right)-\frac{2^{t+3} \log [8]}{\left(76 \times 2^{t}-120\right)}-\frac{\left(40+2^{t}\right) \log [20]}{\left(76 \times 2^{t}-120\right)}-\frac{\left(2^{t+6}-2^{t+4}-160\right) \log [64]}{\left(76 \times 2^{t}-120\right)} . \\
& \text { 3.2.3. The Atom Bond Connectivity Entropy. Now, using } A B C(\mathscr{C} \mathscr{B} \mathscr{T})=\left(\frac{3 \sqrt{2}}{4}+\frac{3 \sqrt{6}}{8}\right) 2^{t}+\sqrt{2}\left(1-\frac{5 \sqrt{3}}{4}\right) .
\end{aligned}
$$
Tables 1 and 4, the atom bond connectivity index is calculated in [28] as follows:
We computed $\mathrm{ENT}_{A B C}$ as follows: 


$$
\begin{aligned}
\operatorname{ENT}_{A B C}(\mathscr{C} \mathscr{B} \mathscr{T})= & \log (A B C)-\frac{1}{(A B C)} \sum_{i=1}^{4} \sum_{l m \in E_{i}(\mathscr{C} \mathscr{B} \mathscr{T})}\left[\sqrt{\frac{\wp(l)+\wp(m)-2}{\wp(l) \times \wp(m)}}\right] \log \left[\sqrt{\left.\frac{\wp(l)+\wp(m)-2}{\wp(l) \times \wp(m)}\right]}\right. \\
\operatorname{ENT}_{A B C}(\mathscr{C} \mathscr{B} \mathscr{T})= & \log \left(\left(\frac{3 \sqrt{2}}{4}+\frac{3 \sqrt{6}}{8}\right) 2^{t}+\sqrt{2}\left(1-\frac{5 \sqrt{3}}{4}\right)\right)-\frac{\left(2^{t}+2^{t-1}+2\right) \log [1 / \sqrt{2}]}{((3 \sqrt{2} / 4)+(3 \sqrt{6} / 8)) 2^{t}+\sqrt{2}(1-(5 \sqrt{3} / 4))} \\
& -\frac{\left(2^{t+1}-2^{t-1}-5\right) \log [\sqrt{6} / 4]}{((3 \sqrt{2} / 4)+(3 \sqrt{6} / 8)) 2^{t}+\sqrt{2}(1-(5 \sqrt{3} / 4))} .
\end{aligned}
$$

\section{Corona Product of Complete Hypertree and a Path $\mathscr{C} \mathscr{B} \mathscr{T}(t) \odot P_{n}$}

Let $\widetilde{\mathscr{G}}_{1}=\left(n_{1}, m_{1}\right)$ and $\widetilde{\mathscr{G}}_{2}=\left(n_{2}, m_{2}\right)$ be two graphs, then corona product of these graphs is outlined as the graph acquired by picking one copy of $\widetilde{\mathscr{G}}_{1}$ and $n_{1}$ copies of $\widetilde{\mathscr{G}}_{2}$ and afterwards associating the $i$ th vertex of $\widetilde{\mathscr{G}}_{1}$ with an edge to each vertex in the $i$ th copy of $\widetilde{\mathscr{G}}_{2}$. It develops from the description of the corona product of two graphs that $\left|V \widetilde{\mathscr{G}}_{1} \odot \tilde{\mathscr{G}}_{2}\right|=n_{1}\left(n_{2}+1\right)$ and $\left|E_{\widetilde{\mathscr{G}}_{1} \odot \tilde{\mathscr{G}}_{2}}\right|=m_{1}+n_{1}\left(m_{2}+n_{2}\right)$ (see details in $[28,32])$. It is noted that corona product of two graphs is no commutative. We demonstrate the corona product of hypertree $\mathscr{C} \mathscr{B} \mathscr{T}(t)$ and path $P_{n}$ for $t=3$ and $n=3$ in Figure 2.

Consider the corona product of complete hypertree and path $\mathscr{C} \mathscr{B} \mathscr{T}(3) \odot P_{3}$ in Figure 2 as an illustration to deduce different topological indices and their respective entropies. To describe our main findings, we form a partition of edges of the corona product of hypertree and path $\mathscr{C} \mathscr{B} \mathscr{T}(t) \odot P_{n}$ for $t, n \geq 2$ established on eccentricity of end vertices in seven sets shown in Tables 5 and 6 representing the edge partition of $\mathscr{C} \mathscr{B} \mathscr{T}(t) \odot P_{n}$. Also, if $l m \in \mathscr{G}_{E}$, then $\wp(l)$ and $\wp(m)$ denote the degrees of vertex $l$ and $m$, respectively.

4.1. Eccentricity-Based Entropies of $\mathscr{C} \mathscr{B} \mathscr{T}(t) \odot \mathscr{P}_{n}$. In this segment, we measure the eccentricity-based entropies of the corona product of hypertree and a path $\mathscr{C} \mathscr{B} \mathscr{T}(t) \odot \mathscr{P}_{n}$.

4.1.1. The Fourth Geometric Arithmetic Eccentric Entropy. Now, using Tables 1 and 5, the fourth geometric arithmetic eccentric index is as follows:

$$
\begin{aligned}
{[5] G A_{4}\left(\mathscr{C} \mathscr{B} \mathscr{T}(t) \odot \mathscr{P}_{n}\right)=} & \left(2^{t}+n\right)+(n-1)\left(2^{t+1}-2\right)+\frac{2 n \sqrt{t^{2}}+3 t+2}{2 t+3}+n \times 2^{t+1} \frac{\sqrt{4 t^{2}+2 t}}{4 t+1} \\
& +(n+2) \sum_{k=1}^{t-1} 2^{k+1} \frac{\sqrt{(t+k)^{2}+(t+k)}}{2(t+k)+1} .
\end{aligned}
$$

We computed $\mathrm{ENT}_{G A_{4}}$ as follows:

$$
\begin{aligned}
& \mathrm{ENT}_{G A_{4}}\left(\mathscr{C} \mathscr{B} \mathscr{T}(t) \odot \mathscr{P}_{n}\right)=\log \left(G A_{4}\right)-\frac{1}{\left(G A_{4}\right)} \sum_{i=1}^{7} \sum_{l m \in E_{i}\left(\mathscr{C} \mathscr{B} \mathscr{T}(t) \odot \mathscr{P}_{n}\right)}\left[\frac{2 \sqrt{\varrho(l) \varrho(m)}}{\varrho(l)+\varrho(m)}\right] \log \left[\frac{2 \sqrt{\varrho(l) \varrho(m)}}{\varrho(l)+\varrho(m)}\right] \\
& \operatorname{ENT}_{G A_{4}}\left(\mathscr{C} \mathscr{B} \mathscr{T}(t) \odot \mathscr{P}_{n}\right)=\log \left(G A_{4}\right)-\frac{(n+2)}{G A_{4}} \sum_{k=1}^{t-1} 2^{k+1} \frac{\sqrt{\left(t^{2}+k^{2}+2 t k+t+k\right)}}{2(t+k+1)} \log \left[\sum_{k=1}^{t-1} 2 \frac{\sqrt{\left(t^{2}+k^{2}+2 t k+t+k\right)}}{2(t+k+1)}\right] \\
& -\frac{n \times 2^{t+1} \sqrt{4 t^{2}+2 t}}{\left(G A_{4}\right)(4 t+1)} \log \left[\frac{2 \sqrt{4 t^{2}+2 t}}{4 t+1}\right]-\frac{2 n \sqrt{(t+1)(t+2)}}{\left(G A_{4}\right)(2 t+3)} \log \left[\frac{2 \sqrt{(t+1)(t+2)}}{2 t+3}\right] . \\
& M M_{1}\left(\mathscr{C} \mathscr{B} \mathscr{T}(t) \odot \mathscr{P}_{n}\right)=(16 n t+4 t-2 n-8) \times 2^{t}-4 n t \\
& +9 n-4 t+6 \text {. }
\end{aligned}
$$
as follows: 


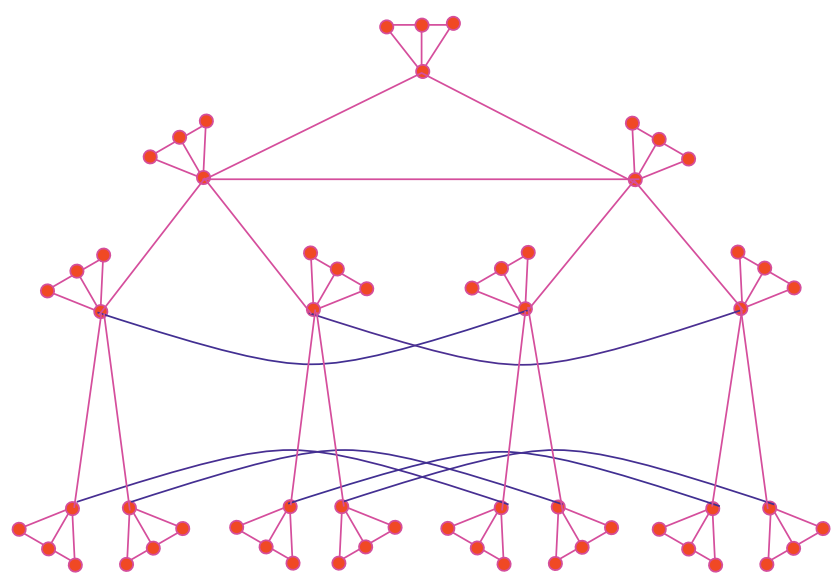

Figure 2: Depiction of corona product of complete hypertree and path $\mathscr{C} \mathscr{B} \mathscr{T}(3) \odot P_{3}[28]$.

TABLe 5: Edge partition of $\mathscr{C} \mathscr{B} \mathscr{T}(t) \odot P_{n}$ grounded on eccentricity of end vertices of every edge, $n \geq 2$.

\begin{tabular}{lcr}
\hline$(\varrho(l), \varrho(m))$ & Frequency & Range of $t$ and $k$ \\
\hline$(t+1, t+1)$ & 3 & $t \geq 2$ \\
$(t+k, t+k+1)$ & $(2+n) 2^{k}$ & $1 \leq k \leq t-1, t \geq 2$ \\
$(2 t, 2 t+1)$ & $2^{t} \times n$ & $1 \leq k \leq t-1, t \geq 2$ \\
$(t+1, t+2)$ & $n$ & $1 \leq k \leq t-1, t \geq 2$ \\
$(t+k+1, t+k+1)$ & $(n-1) 2^{k}$ & $1 \leq k \leq t, t \geq 2$ \\
$(t+k+1, t+k+1)$ & $2^{k}$ & $1 \leq k \leq t-1, t \geq 2$ \\
$(t+2, t+2)$ & $(n-1)$ & $t \geq 2$ \\
\hline
\end{tabular}

TABLE 6: Edge partition of $\mathscr{C} \mathscr{B} \mathscr{T}(t) \odot P_{n}$ for $t, n \geq 2$.

\begin{tabular}{lcc}
\hline$(\wp(l), \wp(m))$ & Frequency & Types of edges \\
\hline$(n+2, n+2)$ & $2^{t-1}$ & $E_{1}$ \\
$(n+2, n+4)$ & $2+2^{t}$ & $E_{2}$ \\
$(n+4, n+4)$ & $\left(2^{t-1}-1\right)+\sum_{j=1}^{t-2} 2^{j+1}$ & $E_{3}$ \\
$(n+2,2)$ & $2\left(2^{t}+1\right)$ & $E_{4}$ \\
$(n+2,3)$ & $(n-2)\left(2^{t}+1\right)$ & $E_{5}$ \\
$(n+4,2)$ & $(n-2) \sum_{j=1}^{t-1} 2^{j}$ & $E_{6}$ \\
$(n+4,3)$ & $2^{t+2}-2$ & $E_{7}$ \\
$(2,3)$ & $(n-3)\left(2^{t}-1\right)$ & $E_{8}$ \\
$(3,3)$ & & $E_{9}$ \\
\hline
\end{tabular}

We computed $\mathrm{ENT}_{M M_{1}}$ as follows:

$$
\begin{aligned}
\mathrm{ENT}_{M M_{1}}\left(\mathscr{C} \mathscr{B} \mathscr{T}(t) \odot \mathscr{P}_{n}\right)= & \log \left(M M_{1}\right)-\frac{1}{\left(M M_{1}\right)} \sum_{i=1}^{7} \sum_{l m \in E_{i}\left(\mathscr{C} \mathscr{B} \mathscr{T}(t) \odot \mathscr{P}_{n}\right)}[\varrho(l)+\varrho(m)] \log [\varrho(l)+\varrho(m)], \\
\mathrm{ENT}_{M M_{1}}\left(\mathscr{C} \mathscr{B} \mathscr{T}(t) \odot \mathscr{P}_{n}\right)= & \log \left((16 n t+4 t-2 n-8) \times 2^{t}-4 n t+9 n-4 t+6\right)-\frac{6(t+1) \log [2 t+2]}{\left(M M_{1}\right)} \\
& -\frac{(n+2)}{\left(M M_{1}\right)}\left((4 t-3) 2^{t}+2-4 t\right) \log \left[3 t^{2}-2 t-1\right]-\frac{n \times 2^{t}}{\left(M M_{1}\right)}(4 t+1) \log [4 t+1] \\
& -\frac{n(2 t+3)}{\left(M M_{1}\right)} \log [2 t+3]-\frac{(n-1)}{\left(M M_{1}\right)}\left(8 t \times 2^{t}-4 t\right) \log \left[(t+1)^{2}+2 t(t+1)-t-1\right] \\
& -\frac{(4 t-2) 2^{t}-4 t}{\left(M M_{1}\right)} \log \left[3 t^{2}-t-2\right]-\frac{(n-1)}{\left(M M_{1}\right)}(2 t+4) \log [2 t+4] .
\end{aligned}
$$


4.1.3. The Second Zagreb Eccentric Entropy. The second Zagreb eccentric index by using Tables 1 and 5 is calculated in [28] as follows:

$$
M M_{2}\left(\mathscr{C} \mathscr{B} \mathscr{T}(t) \odot \mathscr{P}_{n}\right)=\left(16 n t^{2}-4 n t+4 t^{2}+8 n-16 t+7\right) 2^{t}\left(-2 n t^{3}+9 n t-2 t^{2}-2 n+6 t-9\right)
$$

We computed $\mathrm{ENT}_{\mathrm{MM}_{2}}$ as follows:

$$
\begin{aligned}
\mathrm{ENT}_{M M_{2}}\left(\mathscr{C} \mathscr{B} \mathscr{T}(t) \odot \mathscr{P}_{n}\right)= & \log \left(M M_{2}\right)-\frac{1}{\left(M M_{2}\right)} \sum_{i=1}^{7} \sum_{l m \in E_{i}\left(\mathscr{C} \mathscr{B} \mathscr{T}(t) \odot \mathscr{P}_{n}\right)}[\varrho(l) \times \varrho(m)] \log [\varrho(l) \times \varrho(m)], \\
\mathrm{ENT}_{M M_{2}}\left(\mathscr{C} \mathscr{B} \mathscr{T}(t) \odot \mathscr{P}_{n}\right)= & \log \left(M M_{2}\right)-\frac{3(t+1)^{2}}{\left(M M_{2}\right)} \log \left[(t+1)^{2}\right]-\frac{n \times 2^{t+1}}{\left(M M_{2}\right)}\left(2 t^{2}+t\right) \log \left[4 t^{2}+2 t\right] \\
& -\frac{2+n}{\left(M M_{2}\right)}\left[\left(4 t^{2}-6 t+4\right) 2^{t}-2 t^{2}+2 t-4\right] \log \left[\frac{7 t^{3}}{3}-\frac{4 t}{3}-t^{2}\right]-\frac{(n-1)}{\left(M M_{2}\right)}(t+2)^{2} \log \left[(t+2)^{2}\right] \\
& -\frac{n\left(t^{2}+3 t+2\right)}{\left(M M_{2}\right)} \log \left[t^{2}+3 t\right]-\frac{(n-1)}{\left(M M_{2}\right)}\left[\left(8 t^{2}+4\right) 2^{t}-2 t^{2}-4\right] \log \left[\frac{7}{3} t^{3}+\frac{9}{2} t^{2}+\frac{13}{2} t\right] \\
& -\frac{1}{\left(M M_{2}\right)}\left[\left(4 t^{2}-4 t+3\right) 2^{t}-2 t^{2}-4\right] \log \left[\frac{7}{3} t^{3}+\frac{1}{2} t^{2}-\frac{11}{6} t-1\right] .
\end{aligned}
$$

4.1.4. Eccentric Atom Bond Connectivity Entropy. The eccentric atom bond connectivity index by using Tables 1 and 5 is calculated in [28] as follows:

$$
\begin{aligned}
A B C_{5}\left(\mathscr{C} \mathscr{B} \mathscr{T}(t) \odot \mathscr{P}_{n}\right)= & \frac{3 \sqrt{2 t}}{t+1}+(2+n) \sum_{k=1}^{t-1} 2^{k} \sqrt{\frac{2 t+2 k-1}{\left(t^{2}+k^{2}+2 t k+t+k\right)}}+n \times 2^{t} \sqrt{\frac{4 t-1}{4 t^{2}+2 t}}+n \times \sqrt{\frac{2 t+1}{t^{2}+3 t+2}} \\
& +(n-1) \sum_{k=1}^{t} 2^{k} \frac{\sqrt{2 k+2 t}}{t+k+1}+\sum_{k=1}^{t-1} 2^{k} \frac{\sqrt{2 k+2 t}}{t+k+1}+(n-1) \frac{\sqrt{2 t+2}}{t+2}
\end{aligned}
$$

We computed $\mathrm{ENT}_{A B C_{5}}$ as follows: 


$$
\begin{aligned}
& \operatorname{ENT}_{A B C_{5}}\left(\mathscr{C} \mathscr{B} \mathscr{T}(t) \odot \mathscr{P}_{n}\right)=\log \left(A B C_{5}\right)-\frac{1}{\left(A B C_{5}\right)} \sum_{i=1}^{7} \sum_{l m \in E_{i}\left(\mathscr{C} \mathscr{B} \mathscr{T}(t) \odot \mathscr{P}_{n}\right)}\left[\sqrt{\frac{\varrho(l)+\varrho(m)-2}{\varrho(l) \times \varrho(m)}}\right] \log \left[\sqrt{\frac{\varrho(l)+\varrho(m)-2}{\varrho(l) \times \varrho(m)}}\right], \\
& \operatorname{ENT}_{A B C_{5}}\left(\mathscr{C} \mathscr{B} \mathscr{T}(t) \odot \mathscr{P}_{n}\right)=\log \left(A B C_{5}\right)-\frac{3}{\left(A B C_{5}\right)}\left[\frac{\sqrt{2 t}}{t+1}\right] \log \left[\frac{\sqrt{2 t}}{t+1}\right]-\frac{4 \cdot 2^{t}}{\left(A B C_{5}\right)} \cdot \sqrt{\frac{4 t-1}{4 t^{2}+2 t}} \log \left[\sqrt{\frac{4 t-1}{4 t^{2}+2 t}}\right] \\
& -\frac{(2+n)}{\left(A B C_{5}\right)}\left[\sum_{k=1}^{t-1} 2^{k} \sqrt{\frac{2 t+2 k-1}{\left(t^{2}+k^{2}+2 t k+t+k\right)}}\right] \log \left[\sum_{k=1}^{t-1} \sqrt{\frac{2 t+2 k-1}{\left(t^{2}+k^{2}+2 t k+t+k\right)}}\right] \\
& -\frac{n}{\left(A B C_{5}\right)} \sqrt{\frac{2 t+1}{t^{2}+3 t+2}} \log \left[\sqrt{\frac{2 t+1}{t^{2}+3 t+2}}\right]-\frac{(n-1)}{\left(A B C_{5}\right)}\left[\sum_{k=1}^{t} 2^{k} \frac{\sqrt{2 k+2 t}}{t+k+1}\right] \log \left[\sum_{k=1}^{t} \frac{\sqrt{2 k+2 t}}{t+k+1}\right] \\
& -\frac{1}{\left(A B C_{5}\right)}\left[\sum_{k=1}^{t-1} 2^{k} \frac{\sqrt{2 k+2 t}}{t+k+1}\right] \log \left[\sum_{k=1}^{t-1} \frac{\sqrt{2 k+2 t}}{t+k+1}\right]-\frac{(n-1)}{\left(A B C_{5}\right)} \cdot\left[\frac{\sqrt{2 t+2}}{t+2}\right] \log \left[\frac{\sqrt{2 t+2}}{t+2}\right]
\end{aligned}
$$

4.2. Degree-Based Entropies of $\mathscr{C} \mathscr{B} \mathscr{T}(t) \odot \mathscr{P}_{n}$. In this segment, we measure the degree-based entropies of the corona product of hypertree and path $\mathscr{C} \mathscr{B} \mathscr{T}(t) \odot \mathscr{P}_{n}$.
4.2.1. The Hyper Zagreb Entropy. Now, using Tables 1 and 6, the hyper Zagreb index is as follows:

$$
\operatorname{HM}\left(\mathscr{C} \mathscr{B} \mathscr{T}(t) \odot \mathscr{P}_{n}\right)=2\left(n^{3}+34 n^{2}+185 n-66\right) 2^{t}-n^{3}-26 n^{2}-199 n+32
$$

We computed $\mathrm{ENT}_{\mathrm{HM}}$ as follows:

$$
\begin{aligned}
\mathrm{ENT}_{\mathrm{HM}}\left(\mathscr{C} \mathscr{B} \mathscr{T}(t) \odot \mathscr{P}_{n}\right)= & \log (\mathrm{HM})-\frac{1}{(\mathrm{HM})} \sum_{i=1}^{9} \sum_{l m \in E_{i}\left(\mathscr{C} \mathscr{B} \mathscr{T}(t) \odot \mathscr{P}_{n}\right)}[\wp(l)+\wp(m)]^{2} \log \left([\wp(l)+\wp(m)]^{2}\right), \\
\mathrm{ENT}_{\mathrm{HM}}\left(\mathscr{C} \mathscr{B} \mathscr{T}(t) \odot \mathscr{P}_{n}\right)= & \log (\mathrm{HM})-\frac{2^{t-1}}{(\mathrm{HM})}\left((2 n+4)^{2}\right) \log \left[(2 n+4)^{2}\right]-\frac{2+2^{t}}{(\mathrm{HM})}\left[(2 n+6)^{2}\right] \log \left[(2 n+6)^{2}\right] \\
& -\frac{(2 n+8)^{2}}{(\mathrm{HM})}\left(\left(2^{t-1}-1\right) \sum_{j=1}^{t-2} 2^{j+1}\right) \log \left[(2 n+8)^{2}\right]-\frac{(n+4)^{2}}{(\mathrm{HM})}\left(2^{t+1}+2\right) \log \left[(n+4)^{2}\right] \\
& -\frac{(n+5)^{2}}{(\mathrm{HM})}(n-2)\left(2^{t}+1\right) \log \left[(n+5)^{2}\right]-\frac{(n+6)^{2}}{(\mathrm{HM})}\left(2^{t+1}-4\right) \log \left[(n+6)^{2}\right] \\
& -\frac{(n+7)^{2}}{(\mathrm{HM})}(n-2)\left(2^{t}-2\right) \log \left[(n+7)^{2}\right]-\frac{25\left(2^{t+2}-2\right)}{(\mathrm{HM})} \log [25]-\frac{36(n-3)}{(\mathrm{HM})}\left(2^{t+1}-1\right) \log [36] .
\end{aligned}
$$

4.2.2. The Forgotten Entropy. Now, using Tables 1 and 6, the forgotten index is as follows:

$$
F\left(\mathscr{C} \mathscr{B} \mathscr{T}(t) \odot \mathscr{P}_{n}\right)=\left(2 n^{3}+18 n^{2}+114 n-4\right) 2^{t}-n^{3}-18 n^{2}-111 n-82
$$


We computed $\mathrm{ENT}_{F}$ as follows:

$$
\begin{aligned}
\operatorname{ENT}_{F}\left(\mathscr{C} \mathscr{B} \mathscr{T}(t) \odot \mathscr{P}_{n}\right)= & \log (F)-\frac{1}{(F)} \sum_{i=1}^{9} \sum_{l m \in E_{i}\left(\mathscr{C} \mathscr{B}(t) \odot \mathscr{P}_{n}\right)}\left[(\wp(l))^{2}+(\wp(m))^{2}\right] \log \left[(\wp(l))^{2}+(\wp(m))^{2}\right] \\
\mathrm{ENT}_{F}\left(\mathscr{C} \mathscr{B} \mathscr{T}(t) \odot \mathscr{P}_{n}\right)= & \log (F)-\frac{2^{t}}{(F)}(n+2)^{2} \log \left[2(n+2)^{2}\right]-\frac{\left(2+2^{2}\right)}{(F)}\left(2 n^{2}+12 n+20\right) \log \left[2 n^{2}+12 n+20\right] \\
& -\frac{2(n+4)^{2}}{(F)}\left(\left(2^{t-1}-1\right)+\sum_{j=1}^{t-1} 2^{j+1}\right) \log \left[2(n+4)^{2}\right]+\frac{\left(2^{t+1}+2\right)}{(F)}\left(n^{2}+4 n+8\right) \log \left[n^{2}+4 n+8\right] \\
& -\frac{\left(2^{t}+1\right)}{(F)}(n-2)\left(n^{2}+4 n+13\right) \log \left[n^{2}+4 n+13\right]-\frac{\left(2^{t+1}-4\right)}{(F)}\left(n^{2}+8 n+20\right) \log \left[n^{2}+8 n+20\right] \\
& -\frac{(n-2)}{(F)}\left(2^{t}-2\right)\left(n^{2}+8 n+25\right) \log \left[n^{2}+8 n+25\right]-\frac{13}{(F)}\left(2^{t+2}-2\right) \log [13] \\
& -\frac{18}{(F)}(n-3)\left(2^{t+1}-1\right) \log [18] .
\end{aligned}
$$

4.2.3. The Atom Bond Connectivity Entropy. Now, using Tables 1 and 6 , the atom bond connectivity index is as follows:

$$
\begin{aligned}
A B C\left(\mathscr{C} \mathscr{B} \mathscr{T}(t) \odot \mathscr{P}_{n}\right)= & 2^{t-1} \cdot \frac{\sqrt{2 n+2}}{n+2}+\left(2+2^{t}\right) \sqrt{\frac{2}{n+4}}+\left(\left(2^{t-1}+2^{t}-5\right) \frac{\sqrt{2 n+6}}{n+4}+\frac{1}{\sqrt{2}}\left(2^{t+3}-4\right)\right. \\
& +(n-2)\left(2^{t}+1\right) \sqrt{\frac{n+3}{3 n+6}}+(n-2)\left(2^{t}-2\right) \sqrt{\frac{n+5}{3(n+4)}}+\frac{2}{3}(n-3)\left(2^{t+1}-1\right) .
\end{aligned}
$$

We computed $\mathrm{ENT}_{A B C}$ as follows:

$$
\begin{aligned}
& \operatorname{ENT}_{A B C}\left(\mathscr{C} \mathscr{B} \mathscr{T}(t) \odot \mathscr{P}_{n}\right)=\log (A B C)-\frac{1}{(A B C)} \sum_{i=1}^{9} \sum_{l m \in E_{i}\left(\mathscr{C} \mathscr{B} \mathscr{T}(t) \odot \mathscr{P}_{n}\right)}\left[\sqrt{\frac{\wp(l)+\wp(m)-2}{\wp(l) \times \wp(m)}}\right] \log \left[\sqrt{\left.\frac{\wp(l)+\wp(m)-2}{\wp(l) \times \wp(m)}\right]},\right. \\
& \operatorname{ENT}_{A B C}\left(\mathscr{C} \mathscr{B} \mathscr{T}(t) \odot \mathscr{P}_{n}\right)=\log (A B C)-\frac{2^{t-1}}{(A B C)} \cdot \frac{\sqrt{2 n+2}}{n+2} \log \left[\frac{\sqrt{2 n+2}}{n+2}\right]-\frac{\left(2+2^{t}\right)}{(A B C)} \cdot \sqrt{\frac{2}{n+4}} \log \left[\sqrt{\frac{2}{n+4}}\right] \\
& -\frac{\left(2^{t-1}+2^{t}-5\right)}{(A B C)} \cdot \frac{\sqrt{2 n+6}}{n+4} \log \left[\frac{\sqrt{2 n+6}}{n+4}\right]-\frac{\left(2^{t+3}-4\right)}{\sqrt{2}(A B C)} \log \left[\frac{1}{\sqrt{2}}\right]-\frac{2(n-3)\left(2^{t+1}-1\right)}{3(A B C)} \log \left[\frac{2}{3}\right] \\
& -\frac{(n-2)\left(2^{t}+1\right)}{(A B C)} \cdot \sqrt{\frac{n+3}{3 n+6}} \log \left[\sqrt{\frac{n+3}{3 n+6}}\right]-\frac{(n-2)\left(2^{t}-2\right)}{(A B C)} \cdot \sqrt{\frac{n+5}{3(n+4)}} \log \left[\sqrt{\frac{n+5}{3(n+4)}}\right] .
\end{aligned}
$$


TABLE 7: Comparison of degree-based entropies $\mathrm{ENT}_{\mathrm{HM}}, \mathrm{ENT}_{F}$, and $\mathrm{ENT}_{A B C}$ for $\mathscr{C} \mathscr{B} \mathscr{T}(t)$.

\begin{tabular}{lccc}
\hline$[t]$ & $\mathrm{ENT}_{\mathrm{HM}}$ & $\mathrm{ENT}_{F}$ & $\mathrm{ENT}_{\mathrm{ABC}}$ \\
\hline$[2]$ & 2.1246 & 3.5842 & 1.9089 \\
{$[3]$} & 2.9512 & 4.0699 & 2.7883 \\
{$[4]$} & 3.7143 & 4.7145 & 3.5641 \\
{$[5]$} & 4.4418 & 5.3915 & 4.2964 \\
{$[6]$} & 5.1520 & 6.0782 & 5.0086 \\
{$[7]$} & 5.8536 & 6.7685 & 5.7111 \\
{$[8]$} & 6.5509 & 7.4603 & 6.409 \\
{$[9]$} & 7.2462 & 8.1528 & 7.1044 \\
{$[10]$} & 7.9404 & 8.8456 & 7.7987 \\
\hline
\end{tabular}

TABLE 8: Comparison of degree-based entropies $\mathrm{ENT}_{\mathrm{HM}}, \mathrm{ENT}_{F}$, and $\mathrm{ENT}_{A B C}$ for $\mathscr{C} \mathscr{B} \mathscr{T}(t) \odot \mathscr{P}_{n}$.

\begin{tabular}{lccc}
\hline$[n, t]$ & $\mathrm{ENT}_{\mathrm{HM}}$ & $\mathrm{ENT}_{F}$ & $\mathrm{ENT}_{\mathrm{ABC}}$ \\
\hline$[2,2]$ & 3.2403 & 3.2263 & 3.3968 \\
{$[3,3]$} & 4.4033 & 4.1609 & 4.5576 \\
{$[4,4]$} & 5.3793 & 5.0322 & 5.5605 \\
{$[5,5]$} & 6.3577 & 6.0125 & 6.4837 \\
{$[6,6]$} & 7.2580 & 6.8099 & 7.3601 \\
{$[7,7]$} & 7.9193 & 7.6030 & 8.2058 \\
{$[8,8]$} & 8.7118 & 8.3501 & 9.0301 \\
{$[9,9]$} & 9.4911 & 9.0638 & 9.8386 \\
{$[10,10]$} & 10.2609 & 10.0376 & 10.6349 \\
\hline
\end{tabular}

\section{Physical Interpretation of Computed Results}

In QSPR/QSAR deliberations, topological indices are utilized to associate the biological functions of the frameworks with their substantial properties like distortion, strain energy, stability, and melting point [33]. These assessments can be executed by using degree-based indices because these indices have clarity of decision and rapidity [34]. In this section, we talked about some degree-based entropies. We proposed a new approach to estimate the entropy by estimating its topological indices. The forgotten and the hyper Zagreb indices are employed to form the physico-chemical characteristics such as density, volume, entropy, and acentric factor of the underlying structure [35]. The degreebased entropy can also be employed to structural chemistry, social network, biology, ecological networks, and national security. Entropy function is monotonic as in all situations. It can be viewed from Tables 7 and 8 . These numerical tables show the behaviours of the computed results. The graphical representation of these results is observed in Figures 3 and 4 .

Numerous employment of complex networks stranded on the entropy correlated with structural information were issued. In $[36,37]$, many algorithms were recommended to examine the structural complexity. However, the entropy approach is reviewed to be the most substantial approach to distinguish biological networks. Furthermore, eccentricitybased indices have vigorous role due to having the potential of computing pharmaceutical properties. Therefore, we have listed mathematically some eccentricity-based entropies for little considerations of parameters for $\mathscr{C} \mathscr{B} \mathscr{T}(t)$ and $\mathscr{C} \mathscr{B} \mathscr{T}(t) \odot \mathscr{P}_{n}$. Also, we produce tables with the help of Matlab for small estimations for eccentricity of $\mathscr{C} \mathscr{B} \mathscr{T}(t)$ and $\mathscr{C} \mathscr{B} \mathscr{T}(t) \odot \mathscr{P}_{n}$. From Tables 9 and 10, we can note that all the evaluation of entropy are in growing request as the

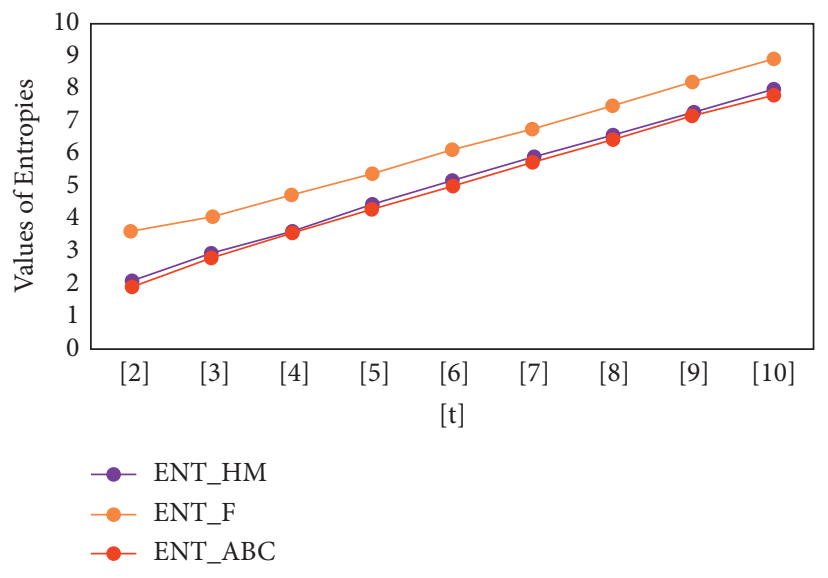

Figure 3: Comparison of entropies for hypertree $\mathscr{C} \mathscr{B} \mathscr{T}(t)$.

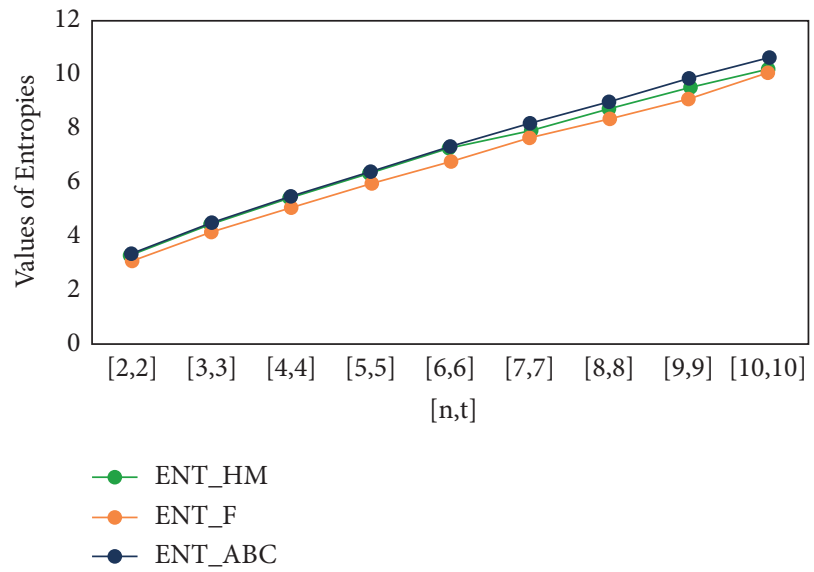

FIGURE 4: Comparison of entropies for corona product of hypertree and a path $\mathscr{C} \mathscr{B} \mathscr{T}(t) \odot \mathscr{P}_{n}$. 
TABLE 9: Comparison of eccentricity-based entropies $\mathrm{ENT}_{G_{4}}, \mathrm{ENT}_{M M_{1}}, \mathrm{ENT}_{M M_{2}}$, and $\mathrm{ENT}_{A B C_{5}}$ for $\mathscr{C} \mathscr{B} \mathscr{T}(t)$.

\begin{tabular}{lcccc}
\hline$[t]$ & $\mathrm{ENT}_{\mathrm{GA}_{4}}$ & $\mathrm{ENT}_{\mathrm{MM}_{1}}$ & $\mathrm{ENT}_{\mathrm{MM}_{2}}$ & $\mathrm{ENT}_{\mathrm{ABC}_{5}}$ \\
\hline$[3]$ & 2.6501 & 2.6731 & 2.4438 & 2.4468 \\
{$[4]$} & 3.1245 & 3.0546 & 2.8593 & 2.7632 \\
{$[5]$} & 3.6393 & 3.5072 & 3.3454 & 3.1550 \\
{$[6]$} & 4.1866 & 4.0074 & 3.8726 & 3.6051 \\
{$[7]$} & 4.7584 & 4.5401 & 4.4264 & 4.0983 \\
{$[8]$} & 5.3482 & 5.0959 & 4.9987 & 4.6234 \\
{$[9]$} & 5.9518 & 5.6689 & 5.5847 & 5.1722 \\
{$[10]$} & 6.5661 & 6.2552 & 6.1815 & 5.7392 \\
{$[11]$} & 7.1887 & 6.8520 & 6.7869 & 6.3204 \\
{$[12]$} & 7.8182 & 7.4574 & 7.3995 & 6.9129 \\
\hline
\end{tabular}

TABle 10: Comparison of eccentricity-based entropies $\mathrm{ENT}_{G A_{4}}, \mathrm{ENT}_{M M_{1}}, \mathrm{ENT}_{M M_{2}}$, and $\mathrm{ENT}_{A B C_{5}}$ for $\mathscr{C} \mathscr{B} \mathscr{T}(t) \odot \mathscr{P}$.

\begin{tabular}{lcccc}
\hline$[n, t]$ & $\mathrm{ENT}_{\mathrm{GA}_{4}}$ & $\mathrm{ENT}_{\mathrm{MM}_{1}}$ & $\mathrm{ENT}_{\mathrm{MM}_{2}}$ & $\mathrm{ENT}_{\mathrm{ABC}_{5}}$ \\
\hline$[2,2]$ & 3.2173 & 3.0186 & 3.5377 & 3.2669 \\
{$[3,3]$} & 4.0058 & 3.528 & 4.7179 & 3.9725 \\
{$[4,4]$} & 4.7234 & 4.001 & 5.7649 & 4.6522 \\
{$[5,5]$} & 5.4110 & 4.4637 & 6.7267 & 5.3390 \\
{$[6,6]$} & 6.0790 & 4.9178 & 7.6328 & 6.0344 \\
{$[7,7]$} & 6.7318 & 5.3632 & 8.5012 & 6.7358 \\
{$[8,8]$} & 7.3716 & 5.8004 & 9.3427 & 8.4406 \\
{$[9,9]$} & 8.0004 & 6.2299 & 10.1644 & 8.1912 \\
{$[10,10]$} & 8.6197 & 6.6524 & 10.9709 & 8.8548 \\
\hline
\end{tabular}

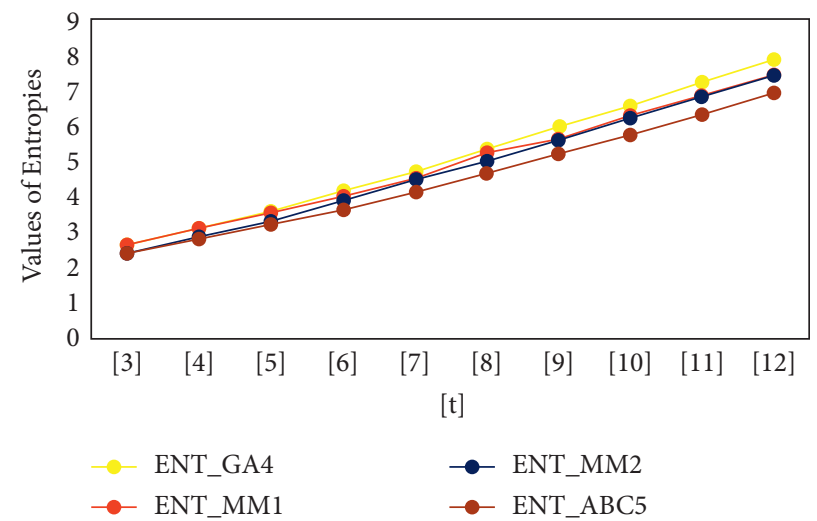

FIGURE 5: Graphical comparison of eccentricity-based entropies for hypertree $\mathscr{C} \mathscr{B} \mathscr{T}(t)$.

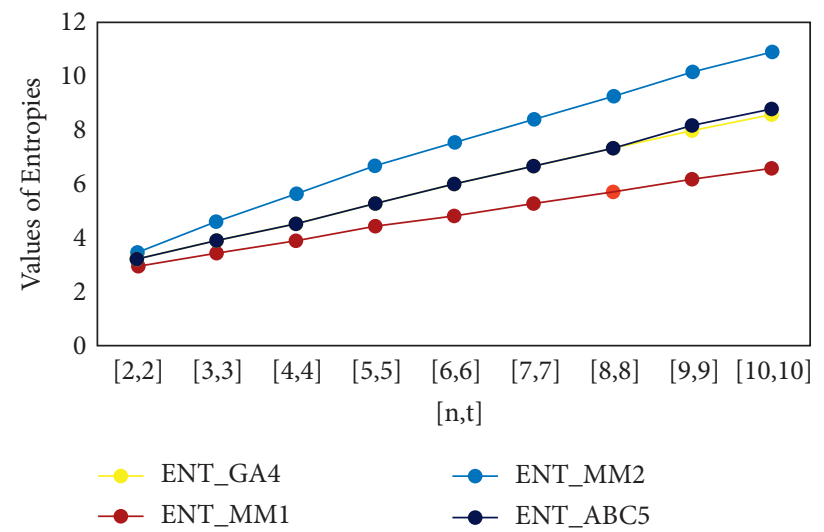

FIGURE 6: Graphical comparison of eccentricity-based entropies for corona product of hypertree and a path $\mathscr{C} \mathscr{B} \mathscr{T}(t) \odot \mathscr{P}$. 
values of parameters are expanded. The graphical representation of computed findings is demonstrated in Figures 5 and 6 for certain measurements of $n$ and $t$.

\section{Conclusion}

In this paper, we have acquired some degree-based and eccentricity-based indices for the depiction of the specific graph theoretical system of biochemical concern. We have acquired aforementioned topological indices for several $t$ level hypertrees and corona product of hypertrees and path. We have also computed the respective entropies. These entropies associate particular physico-chemical characteristics like distortion, stability, melting points, and strain energy of chemical compounds. The mathematical findings for these graphs are helpful for the chemist to understand the biochemical utilization of these structures.

\section{Data Availability}

The data used to support the findings of this study are cited at relevant places within the text as references.

\section{Conflicts of Interest}

The authors declare that they have no conflicts of interest.

\section{Authors' Contributions}

This work was equally contributed by all authors.

\section{References}

[1] B. H. Junker and F. Schreiber, Analysis of Biological Networks, John Wiley \& Sons, Hoboken, NJ, USA, First edition, 2011.

[2] G. A. Pavlopoulos, M. Secrier, C. N. Moschopoulos et al., "Using graph theory to analyze biological networks," BioData Mining, vol. 4, no. 1, pp. 10-27, 2011.

[3] A. Rauf, M. Ishtiaq, M. K. Siddiqui, and R. Andleeb, “Topological properties of doxorubicin conjugated PEG-PAsp copolymer molecular structure used in cancer treatment," Polycyclic Aromatic Compounds, vol. 11, pp. 1-14, 2020.

[4] M. Koutrouli, E. Karatzas, D. Paez-Espino, and G. A. Pavlopoulos, "A guide to conquer the biological network era using graph theory," Frontiers in Bioengineering and Biotechnology, vol. 8, pp. 34-44, 2020.

[5] O. Mason and M. Verwoerd, "Graph theory and networks in biology," IET Systems Biology, vol. 1, no. 2, pp. 89-119, 2007.

[6] M. P. Samanta and S. Liang, "Predicting protein functions from redundancies in large-scale protein interaction networks," Proceedings of the National Academy of Sciences, vol. 100, no. 22, pp. 12579-12583, 2003.

[7] S. Eubank, H. Guclu, V. S. Anil Kumar et al., "Modelling disease outbreaks in realistic urban social networks," Nature, vol. 429, no. 6988, pp. 180-184, 2004.

[8] A. Schnitzler and J. Gross, "Normal and pathological oscillatory communication in the brain," Nature Reviews Neuroscience, vol. 6, no. 4, pp. 285-296, 2005.

[9] O. Diekmann, H. Heesterbeek, and T. Britton, "Understanding infectious disease dynamics," Princeton Series in Theoretical and Computational Biology, Princeton University Press, Princeton, NJ, USA, 2013.
[10] A. J. Kucharski, T. W. Russell, C. Diamond et al., "Early dynamics of transmission and control of COVID-19: a mathematical modeling study," The Lancet Infectious Diseases, vol. 20, no. 5, pp. 553-558, 2020.

[11] K. Roosa, Y. Lee, R. Luo et al., "Real-time forecasts of the COVID-19 epidemic in China from February 5th to February 24th, 2020," Infectious Disease Modeling, vol. 5, pp. 256-263, 2020.

[12] Z. S. Y. Wong, J. Zhou, and Q. Zhang, "Artificial intelligence for infectious disease big data analytics," Infection, Disease \& Health, vol. 24, no. 1, pp. 44-48, 2019.

[13] B. Wang, Y. Sun, T. Q. Duong, L. D. Nguyen, and L. Hanzo, "Risk-aware identification of highly suspected covid-19 cases in social iot: a joint graph theory and reinforcement learning approach," IEEE Access, vol. 8, pp. 115655-115661, 2020.

[14] S. Lalmuanawma, J. Hussain, and L. Chhakchhuak, "Applications of machine learning and artificial intelligence for Covid-19 (SARS-CoV-2) pandemic: a review," Chaos, Solitons \& Fractals, vol. 139, Article ID 110059, 2020.

[15] C. E. Shannon, "A mathematical theory of communication," Bell System Technical Journal, vol. 27, no. 3, pp. 379-423, 1948.

[16] M. Dehmer and M. Graber, "The discrimination power of molecular identification numbers revisited," MATCH Communications in Mathematical and in Computer Chemistry, vol. 69, pp. 785-794, 2013.

[17] Z. Chen, M. Dehmer, and Y. Shi, "A note on distance-based graph entropies," Entropy, vol. 16, no. 10, pp. 5416-5427, 2014.

[18] S. Manzoor, M. K. Siddiqui, and S. Ahmad, "On entropy measures of molecular graphs using topological indices," Arabian Journal of Chemistry, vol. 13, no. 8, pp. 6285-6298, 2020.

[19] S. Manzoor, M. K. Siddiqui, and S. Ahmad, "On physical analysis of degree-based entropy measures for metal-organic superlattices," The European Physical Journal Plus, vol. 136, no. 3, pp. 1-22, 2021.

[20] M. Ghorbani and A. Khaki, "A note on the fourth version of geometric-arithmetic index," The Optoelectronics and Advanced Materials-Rapid Communications, vol. 4, no. 12, pp. 2212-2215, 2010.

[21] M. R. Farahani, "Eccentricity version of atom-bond connectivity index of benzenoid family ABC5 (Hk)," World Applied Sciences Journal, vol. 21, no. 9, pp. 1260-1265, 2013.

[22] G. H. Shirdel, H. RezaPour, and A. M. Sayadi, "The hyper zagreb index of graph operations," Iranian Journal of Mathematical Chemistry, vol. 4, no. 2, pp. 213-220, 2013.

[23] S. Manzoor, M. K. Siddiqui, and S. Ahmad, "Degree-based entropy of molecular structure of hyaluronic acid-curcumin conjugates," The European Physical Journal Plus, vol. 136, no. 1, pp. 1-21, 2021.

[24] S. Manzoor, M. K. Siddiqui, and S. Ahmad, "On entropy measures of polycyclic hydroxychloroquine used for novel coronavirus (COVID-19) treatment," Polycyclic Aromatic Compounds, vol. 8, pp. 1-26, 2020.

[25] S. Manzoor, Y.-M. Chu, M. K. Siddiqui, and S. Ahmad, "On topological aspects of degree based entropy for two carbon nanosheets," Main Group Metal Chemistry, vol. 43, no. 1, pp. 205-218, 2020.

[26] B. Furtula and I. Gutman, "A forgotten topological index," Journal of Mathematical Chemistry, vol. 53, no. 4, pp. 11841190, 2015.

[27] E. Estrada, L. Torres, L. Rodríguez, and I. Gutman, “An atombond connectivity inde. modelling the enthalpy of formation of alkanes," Indian Journal of Chemistry, vol. 37A, pp. 849$855,1998$. 
[28] R. S. Rajan, K. J. Kumar, A. A. Shantrinal, T. M. Rajalaxmi, I. Rajasingh, and K. Balasubramanian, "Biochemical and phylogenetic networks-I: hypertrees and corona products," Journal of Mathematical Chemistry, vol. 59, no. 3, pp. 676698, 2021.

[29] K. Balasubramanian, "Nested wreath groups and their applications to phylogeny in biology and cayley trees in chemistry and physics," Journal of Mathematical Chemistry, vol. 55, no. 1, pp. 195-222, 2017.

[30] M. Arockiaraj, S. R. J. Kavitha, S. Mushtaq, and K. Balasubramanian, "Relativistic topological molecular descriptors of metal trihalides," Journal of Molecular Structure, vol. 12 , pp. $128-138,2020$.

[31] K. Balasubramanian and P. Y. Feng, "Potential-energy surfaces for $\mathrm{Pt} 2+\mathrm{H}$ and $\mathrm{Pt}+\mathrm{H}$ interactions," The Journal of Chemical Physics, vol. 92, no. 1, pp. 541-550, 1990.

[32] R. S. Rajan, A. A. Shantrinal, K. J. Kumar, T. M. Rajalaxmi, I. Rajasingh, and K. Balasubramanian, "Biochemical and phylogenetic networks-II: X-trees and phylogenetic trees," Journal of Mathematical Chemistry, vol. 59, no. 3, pp. 699718, 2021.

[33] Z. Shao, M. Siddiqui, and M. Muhammad, "Computing zagreb indices and zagreb polynomials for symmetrical nanotubes," Symmetry, vol. 10, no. 7, pp. 244-260, 2018.

[34] W. Gao, M. Siddiqui, M. Naeem, and N. Rehman, "Topological characterization of carbon graphite and crystal cubic carbon structures," Molecules, vol. 22, no. 9, pp. 1496-1515, 2017.

[35] A. Alameri, "Second hyper-zagreb index of titania nanotubes and their applications," IEEE Access, vol. 9, pp. 9567-9571, 2021.

[36] G. Bianconi, "The entropy of randomized network ensembles," Europhysics Letters, vol. 81, no. 2, pp. 280-295, 2008.

[37] Y.-H. Xiao, W.-T. Wu, H. Wang, M. Xiong, and W. Wang, "Symmetry-based structure entropy of complex networks," Physica A: Statistical Mechanics and Its Applications, vol. 387, no. 11, pp. 2611-2619, 2008. 